\author{
Military Technical College \\ Kobry El-Kobbah, \\ Cairo, Egypt.
}

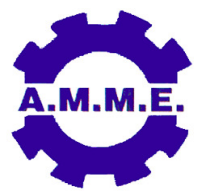

$16^{\text {th }}$ International Conference
on Applied Mechanics and
Mechanical Engineering.

\title{
CONCEPT, THEORY, DESIGN AND EXPERIMENTAL TESTS OF SELF- POWERED LUBRICATION IN CERAMIC COMPOSITE AND ROTORS
}

\author{
J. H. Kim ${ }^{*}$, H. L. Jang ${ }^{* *}$ K. T. Nam ${ }^{* * *}$ and J. Xu ${ }^{* * *}$
}

\begin{abstract}
Due to a modulus of elasticity higher than that of high carbon chrome bearing steel, ceramic rolling elements exhibit less deformation at contact points and therefore a greater stress under the same load. Combined with a lower thermal conductivity, their working temperature increases and their load capacity decreases more rapidly in operation. In Nature, we have a ceramic composite system, Bone, known for its superior load-bearing capacity, and its self-protection at the high-stress movingcontact points (joints) with built-in lubrication. Less known but no less important is the fact that it has a built-in capillary networks for self-powered supply of lubricants and coolants, as well as nutrient and growth factor, from within. It has served as an inspiration to an effort and a model system for the study we report here that aim to Incorporate some of these functionalities into man-made composite structures. In this report, we first highlight our attempt to develop a method for generating networks of micro- and nano-capillaries within a ceramic composite structure during the sintering process. We then present test results of self-powered supply of fluids to the contact (load-bearing) surface via the capillary networks from the fluid reservoir. As a further extension, a self-regulation mechanism is added into the design to enable temperature-controlled self-powered lubrication, and tested in a model system. The method is adaptable to various structural shapes, and scalable in size, and to both biophysiologic and mechanic composite systems.
\end{abstract}

\section{KEY WORDS}

Ceramic rotors and engines, composite, phase-change, bio-mechanics, lubrication

\footnotetext{
* Doctoral Researcher, School of Engineering, Brown University, Providence, USA.

** PhD Candidate, Dpt. of Materials Science and Engineering, Seoul National University, Seoul, Republic of Korea.

*** Professor, Dpt. of Materials Science and Engineering, Seoul National University, Seoul, Republic of Korea.

**** Professor, School of Engineering and Dept. of Physics, Brown University, Providence, USA.
} 


\section{INTRODUCTION}

Nature designs circulatory systems based on hierarchically organized networks of gradually tapered channels. In most biological systems, fluids and nutrients are constantly supplied through such micor-/nano-porous networks by capillary forces and/or osmosis [1]. As the primary structural material of body, bone is a representative of these porous composite systems and affords the body not only a superior load-bearing capability but also self-sustained growth, healing, and lubrication. Ceramic load-bearing composites are now being used in rotors and valves for high-temperature, light-weight, and corrosion-resistant applications. Unlike bones, they are designed and structured mainly for load-bearing in these mechanical applications. However, there have been separate biomedical engineering pursuits for composite materials and structures, e.g. synthetic HAP preparation, for bio-physicochemical in vitro and in vivo medical applications such as middle ear implants, reconstructive joint replacement, and bone implants etc.). [2-4]. It seems only natural for the developments in the two fields to cross paths and benefit from each other's advances. In this work, we report on our findings from an early phase exploration of the Concept, Theory, Design, and Experimental Tests of Self-Powered Lubrication in Ceramic Composite and Rotors, as part of a Korea-USA bilateral research program, supported by NRF of Korea and AOARD of USA.

All the key elements of this exploration, the concept, theory, design, and experimental tests of self-powered lubrication, are shared by the interests in developing next generation functional composites for both biophysiologic and mechanical structures. In experimental tests of the concept, theory, and design reported here, we have focused on one composite material as a model platform that can tie the interests on both fronts: Porous hydroxyapatite [HAP: $\mathrm{Ca}_{10}\left(\mathrm{PO}_{4}\right)_{6}(\mathrm{OH})_{2}$ ]. HAP composites are compounds of great interest in an interdisciplinary field of sciences involving chemistry, biology, medicine, physics, and geology. The findings are generally applicable to other ceramic composites.

\section{CONCEPT AND DESIGN}

HAP is the most stable calcium phosphate salt at normal temperatures and $\mathrm{pH}$ between 4 and 12. It has many interesting applications such as catalysis, fertilizers and pharmaceutical, protein chromatography, water treatment processes, preparation of biocompatible materials, and mostly because it is the main inorganic component in calcified hard tissues (e.g., bone and teeth) of vertebrates [5, 6]. Also HAP is formed pathologically as a result of functional irregularities resulting in cartilage arthritis, formation of renal, bladder, and bile stones, atheromatic plaque, and calcification of transplanted cardiac valves [7]. As such, HAP has been employed as a model compound to study bio-mineralization phenomena [8-11]. As the primary structural material of the body, bone tissue must withstand a variety of static and dynamic loading conditions. Same can be said of ceramic rotors. Bone tissue is a composite of tough, though flexible, collagen fibers reinforced with calcium phosphate nanocrystals. The composite structure of bone gives it much greater stiffness than typical tissues while at the same time providing surprisingly high fracture toughness and resistance to damage, especially compared to other biological tissues [12]. As a living tissue bone reacts to physical stimuli, growing in 
response to imposed loads, and capable of detecting damaged (but not yet completely fractured) areas of tissue and replacing them through a process called remodeling.

Tissue and organ failures resulting from trauma, disease, aging, or frictional movements in case of joints, have been reported to account for half of the annual healthcare expenditures in the US [13]. Current treatments such as transplantation of tissues and organs, surgical repair, the use of artificial prostheses or mechanical devices, and drug therapy are effective but suffer from limitations [13]. Autografts with optimal osteoconductive, osteoinductive, and osteogenic properties are known as best method for bone repair, [14] despite the disadvantages of donor site morbidity and limited availability. Alternative bone allografts suffer from possible transmission of diseases, immune reaction, and uncertain healing to bone. Tissue engineering approaches using an osteoconductive scaffold loaded with growth factors and osteogenic cells have shown great potential for bone repair and regeneration $[13,15]$.

In many ways, Bone implant should satisfy a larger set and often more stringent requirements than ceramic parts in rotors and valves. Ideally, the implant should be biocompatible, bioactive, or biodegradable [16]. The implant should have a porous three-dimensional architecture capable of supporting cell and tissue infiltration, transport of nutrients, and development of capillaries [17]. It must have the requisite mechanical properties for supporting loads experienced by the bone to be replaced [18]. Considerable improvements in scaffold design, fabrication, and evaluation are necessary to meet these stringent requirements, particularly for applications in the repair of load-bearing bone.

Like the moving parts in a ceramic rotor or valve, bone joints are lubricated. They, a marvel of engineering, are structured to maintain their lubrication with lubricin - a principal boundary lubricating and anti-adhesion protein found in a thick colorless liquid that surrounds joints (synovial fluid). These fluids are self-supplied over the parts of the joint not reached by blood vessels that supply nutrition and lubrication, allowing the bones to glide over each other. However this self-lubrication mechanism could be disrupted, damaged, or simply absent, for example in cases of arthritis or implants. Compounding the problem in the latter, much evidence has been found that fretting is a potential degradation failure mode for orthopedic implants [19-22]. Shearing micro-movements may often appear at the interface between the implant and bone due to a large difference in elastic modulus of the two materials in contact. Insufficient initial fixing prosthesis design problem, or the movement of the limb which sustains a large number of stress reversals in the course of 1 day can also cause micro movement $[23,24]$. The oscillatory micro-movements at the contact induce fretting wear, fretting corrosion and sometimes, fatigue cracks, causing the early failure of joint prosthesis $[25,26]$. Thermal sprayed calcium phosphate coatings have been actively studied and positive results of encouraging bone growth have been shown from either in vitro or in vivo testing [27, 28]. Previous work has investigated the fretting behaviors of plasma sprayed hydroxyapatite_HA.bioceramic coatings under unlubricated condition and the results showed the poor fretting wear resistance of HA coatings due to the lamellar structure, high porosity, poor cohesion and adhesion strength, etc [29, 30]. Nevertheless, bone grafts are necessary in orthopaedic surgery for filling bone cavities, treatment of nonunion and replacement 
of bone lost during trauma and tumor removal. Incorporating fluidic, nutrient, and lubricant supply and management into bone implants would represent a significant step forward in the development. In this regard, porous HAP composite structures also stand out.

HAP has been explored for use as synthetic bone grafts allowing successful tissue in-growth, which further enhances the implant-tissue attachment [31]. Along with gradient structures, defined as materials which have a gradient of properties as found in natural bone [32, 33], we have conducted a series of experiments and tests to show that it is indeed possible to achieve self-powered supply of nutrients and/or lubricants in HAP composite structures.

Implementing the design of porous materials with a porosity gradient mimicking that found in natural bone is a main challenge [34]. In this work, we report on our success, still preliminary, in building gradient capillary networks within a HAP composite, and testing their ability to provide continuous distributed supply of lubricants and healing agents through the load-bearing composites and ceramic engine parts. With the success in HAP composites as a demonstration vehicle, we hope to show that it is feasible to develop self-lubricating ceramic rotors with the concept, design, and theory tested with this model system. The HAP composites were fabricated by a new method taking advantage of the pressure-dependent phase-separation between HAP and polymer (e.g. agarose gel). This new method for fabrication of composite with built-in capillary networks for self-powered fluidic supply has many merits such as being biocompatible, shape conformal, and readily scalable in sizes, etc. With this method, the porosity is easily controlled by changing weight percentage of agarose gel in the composite. A linear or radial gradient of the porosity and pore size can be obtained by stacking HAP-agarose gel composites of gradually changing weight percentage of agarose gel. In experiments we have demonstrated the self-powered lubrication enabled by the built-in capillary force. Moreover, we show that it is possible to extend the self-powered lubrication functionality further to include temperature-controlled self-regulation of the supply.

\section{DESIGN, METHOD, EXPERIMENTALS}

Hydroxyapatite (HAP: $\left.\mathrm{Ca}_{10}\left(\mathrm{PO}_{4}\right)_{6}(\mathrm{OH})_{2}\right)$ was synthesized by the precipitation method with calcium hydroxide $\left(\mathrm{Ca}(\mathrm{OH})_{2}, 99.0 \%\right.$, High Purity Chemical, Japan) and phosphoric acid $\left(\mathrm{H}_{3} \mathrm{PO}_{4}, 85.0 \%\right.$, Junsei Chemical Co., Ltd) in aqueous base [42]. In distilled water, $0.5 \mathrm{M}$ of $\mathrm{Ca}(\mathrm{OH})_{2}$ was prepared. After stirring vigorously with a mechanical stirrer (overhead stirrer MSM-1 Jeio Tech) for an hour, $0.5 \mathrm{M}$ of $\mathrm{H}_{3} \mathrm{PO}_{4}$ was added drop-by-drop using a digital burette (Metrohm 876, Dosimat Plus). The final composition ratio of $\mathrm{Ca}(\mathrm{OH})_{2}$ and $\mathrm{H}_{3} \mathrm{PO}_{4}$ was 10:6 and the precipitants were aged for 24 hours while stirring at room temperature. The HAP solution was filterpressed and freeze-dried. Dried HAP powder was mixed with agarose gel powder (Sigma Aldrich) in DI water and sonicated to make homogeneous colloidal mixture. The colloidal mixture was heated in an oven and cooled down at room temperature. The gel composite was dried and sintered at $1100{ }^{\circ} \mathrm{C}$ for 2 hours. Weight ratio of HAP and agarose gel was 5:1 and $2 \mathrm{wt} \%$ of agarose gel in water in standard mixture. By changing the weight ratio, different porosities and capillary pore sizes can be ascertained in the same sintering process. By stacking HAP-agarose gel 
composites of gradually changing weight percentage of agarose gel, one can then obtain a gradient porous material with built-in networks of capillary channels tapered from one end to the other.

To add a self-regulation mechanism onto the self-powered supply of lubricants or biomolecules, we made use of a phase-changing effect achievable in a number of polymers. One such phase-changing polymer is poly $(\mathrm{N}$-isopropylacrylamide $)$ (PNIPAM) whose phase-change temperature is in the convenient range of $32{ }^{\circ} \mathrm{C}$. $[40,41]$. It changes phase from being hydrophilic to hydrophobic when its temperature is raised above this temperature. In the proof-of-concept tests reported here, the phase-changing temperature is $32 \mathrm{C}$, which itself is adjustable through altering the polymer synthesis process. The phase-change is accompanied with a volume-change from being swelled in its hydrophilic state (below 32C) to half of that volume in its hydrophobic state (above $32 \mathrm{C}$ ). With this phase and volume change the payload (lubricant, coolant, or biomolecules) would be secreted (squeezed) out much like the sweat secretion by sweat gland in human skin. For the tests of this mechanism in the actual ceramic composite structures under development, a composite block obtained from the fabrication process described above was infiltrated with the aqueous mixture solution of PNIPAM $(10 \mathrm{mg} / \mathrm{ml}$, Sigma Aldrich) and fluorescent dye, fluorescein isothiocyanate (FITC) $(0.5 \mathrm{mM}$, Sigma Aldrich) and dried. Its surface was thoroughly rinsed and cleaned to remove any residual of the polymer mixture adhered to the surface during the process. For the self-regulated release (secretion) tests, a sample was first placed into water kept at below $32 \mathrm{C}$. It was observed by inverted fluorescence microscope (Leica) to examine whether PNIPAM/FITC was well incorporated into the nanopores of the composite uniformly and whether any unintended release of the FITC occurred before the release mechanism is activated (by raising temperature). It was subsequently placed in hot water of $60{ }^{\circ} \mathrm{C}$. Released FITC in the water was collected every $5 \mathrm{~min}$ and fluorescence intensity was quantitatively measured using fluorometer (Cary Eclipse).

\section{RESULTS AND DISCUSSIONS}

Microscopic morphology of synthetic composite samples was imaged by scanning electron microscope (SEM) (LEO 1530-vp). Figure 1 shows the SEM image of synthesized HAP in the pre-sintering compressed powder state (Fig. 1a), low magnification (Fig. 1b) and high magnification of the sample after sintering at 1100 ${ }^{\circ} \mathrm{C}$ for $2 \mathrm{hrs}$. As-synthesized HAP powder shows nano-needle-like fluffy powder, which is general characteristic for synthetic method we adopted [42]. The samples after sintering showed micro- and nano- porous channel (capillary) networks, whose pore channel size ranged from $\sim 100 \mathrm{~nm}$ to $\sim 1 \mathrm{um}$ (Fig. 1b and 1c). More detailed structural characterization have been carried out by micro-CT on different samples made in similar sintering processes and confirmed the interconnection of these porous channels into networks of capillaries. Porosity of $45 \%$ was obtained by a water infiltration method, also called imbibitions method [43]. Briefly, volume and weight of original bone were measured, water was infiltrated into bone, and weight of bone/water was measured. From the difference of weight between dried and wetted bone, infiltrated water weight and volume was calculated. More precise porosity will be measured by micro-CT in further study. This HAP/agarose gel method offers many advantages such as biocompatibility, environmental friendliness, easy 
preparation, large scale preparation, etc. Also arbitrary shape is possible with this method including cylinder, square rod, thin plate as small as a micrometer, and hollow tubes mechanically inserted or drilled into the structures, etc. This shape conformity gives great flexibility to make various shapes that would be needed in both bio and mechanical applications. In future study porosity control will be carried out by controlling weight ratio of HAP and agarose and also for gradient material preparation.

In examining whether the PNIPM/FITC was infiltrated into composite structure sample uniformly, by using inverted fluorescence microscope, post-infiltration samples were cut at the middle and fluorescence images were taken from center to edge with $2 \mathrm{~mm}$ spacing. (Fig. 2) Figure $2 \mathrm{a}$ was taken at the center position, Fig. 2b at $2 \mathrm{~mm}$ away from the center, Fig. $2 \mathrm{c}$ at $4 \mathrm{~mm}$ away from the center, and Fig. $2 \mathrm{~d}$ at $6 \mathrm{~mm}$ away from the center. These figures show uniformly distributed green fluorescence throughout whole bone sample and dark spots indicate the solid HAP without fluorescent molecule and PNIPAM. Laser confocal microscope may also be adopted for more detailed analysis of infiltration. Infiltrated bone with PNIPAM/FITC released FITC at temperature above the PNIPAM's phase-changing temperature $32 \mathrm{C}$. For a quantitative measurement of the release rate and kinetics, the sample was kept at $60 \mathrm{C}$ and FITC secreted by the PNIPAM and transported by the capillary force from inside of the composite to the surface into the water was collected every 5 min. The fluorescence intensity was measured using fluorometer (excitation wavelength $=480 \mathrm{~nm}$, Cary Eclipse) (Fig. 3a). For control experiment, FITC release at room temperature was carried out (Fig. 3b, red curve). Fig. 3a shows the spectra of released FITC with time at $60^{\circ} \mathrm{C}$ and Fig. $3 \mathrm{~b}$ is the corresponding plot of peak intensity change with time, in which black curve represents releasing profile at $60^{\circ} \mathrm{C}$ and red curve at room temperature. Phase change- or temperature-activated release (Fig. 3b, red curve) clearly shows regulated and faster release than diffusion, confirming the feasibility of incorporating self-regulation mechanism on top of the self-powered supply of biomolecules, lubricants, coolants, and drugs through the networks of capillaries. The storage (or loading) capacity of the payload (e.g. FITC) of the composite was estimated by calculating the weight difference between raw dried ceramic composite and the infiltrated one with $0.5 \mathrm{mM} \mathrm{FITC}$ and $10 \mathrm{mg} / \mathrm{ml}$ PNIPAM. With a cylindrical sample of diameter $=10 \mathrm{~mm}$ and height $=17 \mathrm{~mm}$, the payload of FITC was 0.35 umol. At $60{ }^{\circ} \mathrm{C}, 10 \%$ of FITC was released in 5 min and $30 \%$ in 40 min, after 40 min the release tapered down. At RT, it was 5\% in 5 min, 9\% in $40 \mathrm{~min}$ and $15 \%$ in $120 \mathrm{~min}$. In further study the releasing profiles above phase changing temperature can be controlled or optimized by changing sample size, porosity, concentration of infiltration solution, surface-grafting of PNIPAM, etc.

\section{CONCLUSION}

In this work, we report on an effort, inspired by the mechanisms and structures found in Natural composite structures (e.g. bone) to enable self-powered and self-regulated lubrication into man-made composites structures. It has applications in both biophysiologic and mechanical domains. In the latter case, it may help address a particular problem in ceramic rotors and engines. Although the problem could be attributed to ceramics' modulus of elasticity being higher than that of high carbon chrome bearing steel, and therefore to the fact that ceramic rolling elements exhibit 
less deformation at contact points and therefore a greater stress under the same load which when combined with a lower thermal conductivity leads to more rapid increase in working temperature and decrease in their load capacity decreases in operation.

In this effort, we explored a new method to prepare the ceramic composite in order to build networks of capillaries into the ceramic structure during the sintering process to enable the self-powered and self-regulated lubrication and other desirable new functionalities without sacrifices its mechanical toughness and load-bearing capavity. A HAP/agarose gel system was used as a model system for the study and for the tests of the new functionalities. This model system and the synthesis and sintering method offer many advantages such as biocompatibility, environmental friendliness, easy preparation, large scale preparation, etc. Also it is possible to structure the ceramic composite into any shape by design, including cylinder, square rod, thin plate as small as a micrometer, rod with small holes, etc. In future studies, porosity control can be achieved by controlling weight ratio of HAP and agarose and also continuously gradient material is an open option.

Self-regulated and self-powered supply of fluids was successfully demonstrated by using the resultant HAP composite infiltrated with phase changing polymers (PNIPAM). Above the phase changing temperature of PNIPAM, the polymer changes its phase from hydrophilic to hydrophobic and thereby secretes its payload such as lubricants, coolants, or healing agents, which would then be drawn up by the capillaries and transported to the designated surface of the composite. The operation mechanism is generally applicable, beyond autonomous lubrication in ceramic rotors and valves, to bio-mechanics, e.g. synthetic joints, skeletal repairs, thermal management of embedded electronics, as well as drugs delivery.

This built-in mechanism of autonomous supply of desired fluids to the designated surface of the ceramic part could complement and be coupled to an external resupply mechanism in the periphery for continued operation. Pulling these mechanisms and components together in one ceramic composite requires understanding the conflicting demands and constraints for which one could resort to both experiments and tests as well as the guidance of theory, on which one could focus in future studies.

\section{REFERENCES}

[1] Bejan, A. and Lorente, S., "Constructal theory of generation of configuration in nature and engineering", J. Appl. Phys., 100, 041301, (2006).

[2] Drucker, D. A., Capello, W. N., D'Antonio, J. A., and Hile, L. A., "Works in progress \#6. Total hip arthroplasty using a hydroxyapatitecoated acetabular and femoral component”, Orthop. Rev., 20, 179-185 (1991).

[3] Deeb, M. E., Hosny, M., and Sharawy, M., "Osteogenesis in composite grafts of allogenic demineralized bone powder and porous hydroxyapatite", J. Oral Maxillofac. Surg., 47, 50, (1989).

[4] Jahn, A. F., "Experimental applications of porous (coralline) hydroxyapatite in middle ear and mastoid reconstruction", Laryngoscope, 102, 289, (1992). 
[5] Christoffersen, J., Christoffersen, M. R., Larsen, R., and Møller I. J., "Regeneration by surface-coating of bone char used for defluoridation of water", Water Res., 25, 227, (1991).

[6] Walsh, W. R. and Guzelsu, N., "Compressive properties of cortical bone: mineral-organic interfacial bonding", Biomaterials, 15, 137, (1994).

[7] Koutsopoulos, S., Kontogeorgou, A., Petroheilos, J., and Dalas, E., "Calcification of porcine and human cardiac valves: testing of various inhibitors for antimineralization", J. Mater. Sci. Mater. Med., 9, 421, (1998).

[8] Nancollas, G. H., "Enamel apatite nucleation and crystal growth", J. Dent. Res., 58B, 861, (1979).

[9] Dalas, E. and Koutsoukos, P. G., "Crystallization of hydroxyapatite from aqueous solutions in the presence of cadmium", J. Chem. Soc. Farad. Trans., 85, 3159, (1984).

[10] Koutsopoulos, S., Demakopoulos, I., Argiriou, X., Dalas, E., Klouras, N., and Spanos, N., "Inhibition of hydroxyapatite formation by zirconocenes", Langmuir 11, 1834, (1995).

[11] Boskey, A. L. and Bullough, P. G., "Cartilage calcification: normal and aberrant”, Scan. Electron Microsc., pt II, 943, (1984).

[12] Weiner, S. and Wagner, H. D., "The material bone: structure-mechanical functions relations”, Ann. Rev. Mater. Sci., 28, 271, (1998).

[13] Persidis, A., "Tissue engineering", Nat. Biotechnol., 17, 508, (1999).

[14] Cypher, T. and Grossman, J., "Biological principles of bone graft healing", J. Foot Ankle Surg., 35, 413, (1996).

[15] Marcacci, M., Kon, E., Moukhachev, V., Lavroukov, A., Kutepov, S., Quarto, R., Mastrogiacomo, M., and Cancedda, R., "Stem Cells Associated with Macroporous Bioceramics for Long Bone Repair: 6- to 7-Year Outcome of a Pilot Clinical Study”, Tissue Eng., 13, 947, (2007).

[16] Giannoudis, P. V., Dinopoulos, H., and Tsiridis, E., "Bone substitutes: An update", Injury, Int. J. Care Injured, 36, S20, (2005).

[17] Hutmacher, D. W., "Scaffolds in tissue engineering bone and cartilage", Biomaterials, 21, 2529, (2000).

[18] Hollister, S. J., "Porous scaffold design for tissue engineering", Nat. Mater., 4, 518, (2005).

[19] Dumbleton, J. H., "Tribology of Natural and Artificial Joints: Tribology Series 3", Elsevier, Amsterdam, 32, (1981).

[20] Kawalec, J. S., Brown, S. A., Payer, J. H., and Merritt, K., "Mixed-metal fretting corrosion of $\mathrm{Ti}_{6} \mathrm{Al}_{4} \mathrm{~V}$ and wrought cobalt alloy", J. Biomed. Mater. Res., 29, 867, (1995).

[21] Gilbert, J. L., Buckley, C. A., and Jacobs, J. J., "In vivo corrosion of modular hip prosthesis components in mixed and similar metal combinations, the effect of crevice, stress, motion and alloy coupling", J. Biomed. Mater. Res., 27, 1522, (1993).

[22] Sivakumar, M., Dhanadurai, K. S. K., and Rajeswari, S., "Failures in stainless steel orthopedic implant devices: a survey", J. Mater. Sci. Lett., 14, 351, (1995).

[23] Walker, P. S., Schneeweis, P., Murphy, S., and Nelson, P., "Strains and micromotions of press-fit femoral stem prostheses", J. Biomechanics, 20, 693, (1987).

[24] Sudarshan, T. S. and Jeandin, M., "Surface Modification Technologies VIII", The Institute of Materials, 43, (1995). 
[25] Hoppner, D. W. and Chandrasekaran, V., "Fretting in orthopedic implants: a review", Wear, 173, 189, (1994).

[26] Lambardi, A. L., Mallory, T. H., Vaughn, B. K., and Drouillard, P., "Aseptic loosening in total hip arthroplasty secondary to osteolysis induced by wear debris from titanium alloy modular heads", J. Bone Joint Surg. Am. B, 71, 1337, (1989).

[27] Cook, S. D., Thomas, K. A., Dalton, J. E., Volkman, T. K., Whitecloud III, T. S., and Kay, J. F., "Hydroxyapatite coating of porous implants improves bone ingrowth and interface attachment strength", J. Biomed. Mater. Res., 26, 989, (1992).

[28] Brossa, F., "Adhesion properties of plasma sprayed hydroxyapatite coatings for orthopedic prostheses", Biomed. Mater. Eng., 3, 127, (1993).

[29] Fu, Y.Q., Batchelor, A.W., Wang, Y., and Khor, K. A., "Fretting wear behavior of thermal sprayed hydroxyapatite (HA) bioceramic coating under unlubricated conditions", Wear, 217, 132, (1998).

[30] O'Nell, P. L. and Stachowiak, G.W., "The lubricating properties of arthritic synovial fluid", The 1st World Congress in Bioengineering, 2, 269, (1996).

[31] Chu, T. M. G., Orton, D. G., Hollister, S. J., Feinberg, S. E., and Halloran, J. W., "Mechanical and in vivo performance of hydroxyapatite implants with controlled architectures", Biomaterials, 23, 1283, (2002).

[32] Kieback, B., Neubrand, A., and Riedel, H., "Processing techniques for functionally graded materials", Mater. Sci. Eng. A, 362, 81, (2003).

[33] Pompe, W., Worch, H., Epple, M., Friess, W., Gelinsky, M., Greil, P., Hempel, U., Scharnweber, D., and Schulte, K., "Functionally graded materials for biomedical applications", Mater. Sci. Eng. A, 362, 40, (2003).

[34] Hing, K. A., Best, S. M., and Bonfield, W., "Characterization of porous hydroxyapatite", J. Mater. Sci. Mater. Med., 10, 135, (1999).

[35] Tampieri, A., Celotti, G., Sprio, S., Delcegliano, A., and Franzese, S., "Porositygraded hydroxyapatite ceramics to replace natural bone", Biomaterials, 22, 1365, (2001).

[36] Arita, I. H., Castano, V. M., and Wilkinson, D. S., "Synthesis and processing of hydroxyapatite ceramic tapes with controlled porosity", J. Mater. Sci. Mater. Med., 6, 19, (1995).

[37] Vaz, L., Lopes, A. B., and Almeida, M., "Porosity control of hydroxyapatite implants", J. Mater. Sci. Mater. Med., 10, 239, (1999).

[38] Werner, J., Linner-Krcmar, B., Friess, W., and Greil, P., "Mechanical properties and in vitro cell compatibility of hydroxyapatite ceramics with graded pore structure", Biomaterials, 23, 4285, (2002).

[39] Carotenuto, G., Spagnuolo, G., Ambrosio, L., and Nicolais, L., "Macroporous hydroxyapatite as alloplastic material for dental applications", J. Mater. Sci.: Mater. Med., 10, 671, (1999).

[40] Graziano, G., "On the temperature-induced coil to globule transition of poly-Nisopropylacrylamide in dilute aqueous solutions", Int. J. Biol. Macromol., 27, 89, (2000).

[41] Jones, C. D. and Lyon, L. A., "Shell-restricted swelling and core compression in poly(N-isopropylacrylamide) core-shell microgels", Macromolecules, 36, 1988, (2003).

[42] Bouyer, E., F. Gitzhofer, F., and Boulos, M. I., "Morphological study of hydroxyapatite", J. Mater. Sci.: Mater. Med., 11, 523, (2000). 
[43] Dullien, F. A. L., "Porous Media. Fluid Transport and Pore Structure", Academic Press, (1992).

\section{ACKNOWLEDGEMENT}

This research was supported by the US-Korea bilateral research programs on BioNano-Info and electronic materials, enabled by the National Research Foundation of Korea and the Asia Office of Aerospace Research and Development, AFOSR, and NSF of USA. 


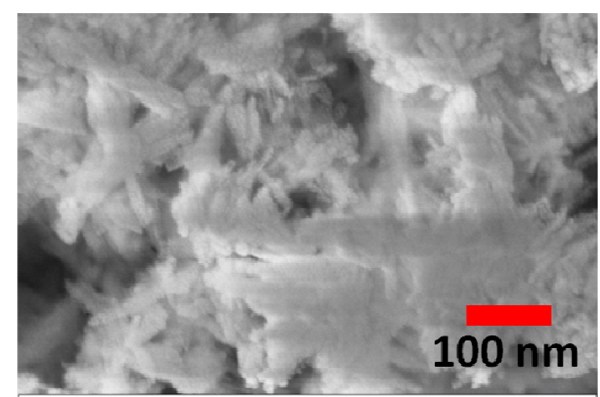

(a)

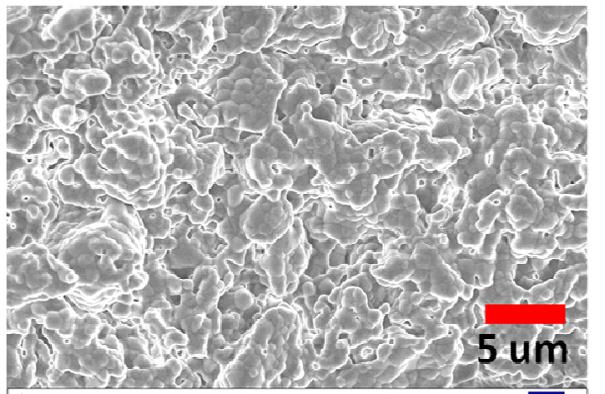

(b)

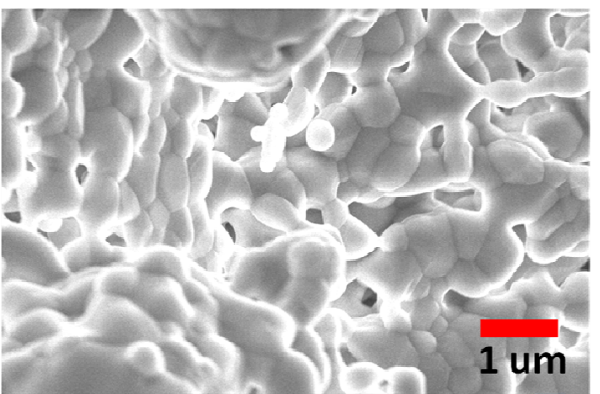

(c)

Fig.1. SEM image of synthetic bone: a) as-synthesized HAP powder, b) low magnification image of synthetic bone, and c) high magnification image of synthetic bone. The weight ratio of HAP and agarose is $5: 1$ and sintered at $1100^{\circ} \mathrm{C}$ for $2 \mathrm{hrs}$.

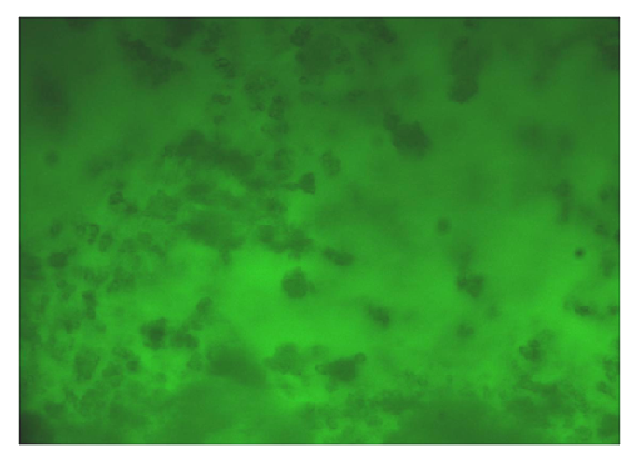

(a)

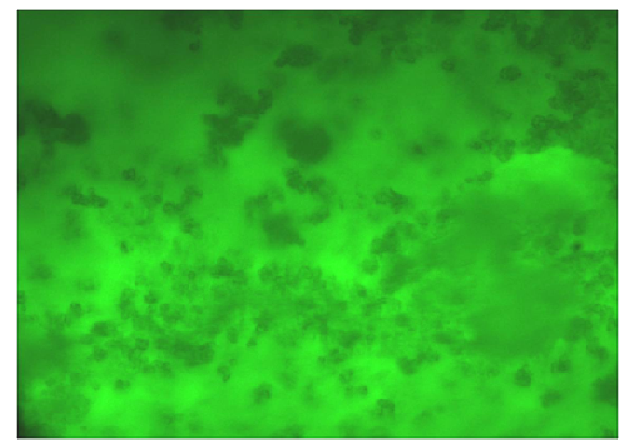

(c)

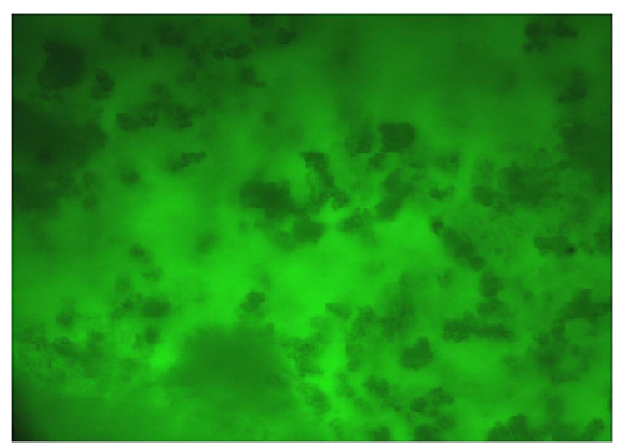

(b)

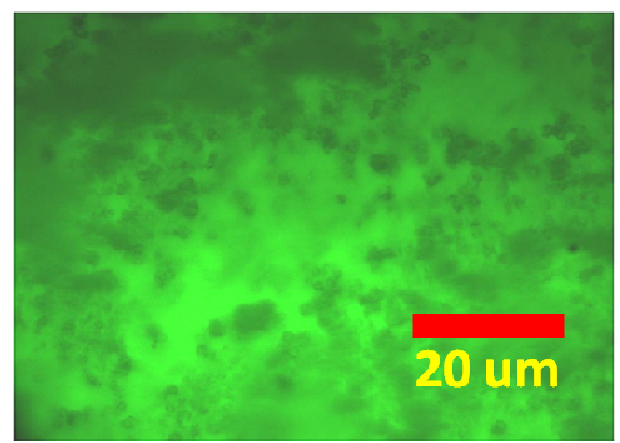

(d)

Fig. 2. Cross-sectional fluorescence image of synthetic bone infiltrated with PNIPAM/FITC: a) center position, b) $2 \mathrm{~mm}$, c) $4 \mathrm{~mm}$, and d) $6 \mathrm{~mm}$ away from center. 


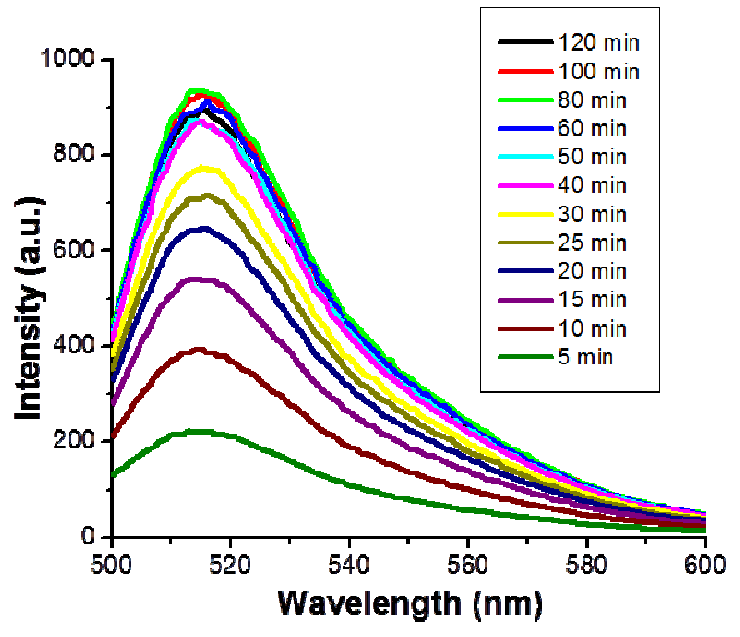

(a)

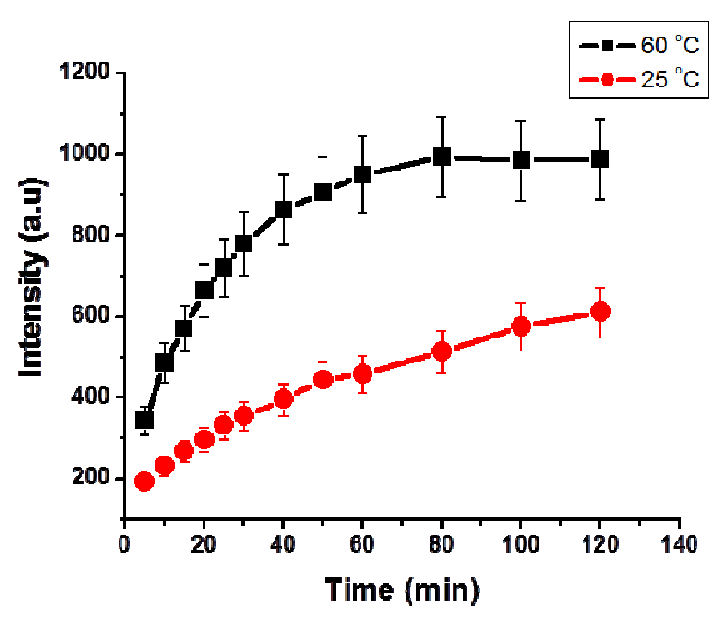

(b)

Fig. 3. Releasing profile of FITC from synthetic bone infiltrated with PNIPAM/FITC:

a) fluorescence spectra taken at $60^{\circ} \mathrm{C}$ and b) peak intensity change with time,

b) black curve from $60^{\circ} \mathrm{C}$ and red one from room temperature. 